\title{
Detailed study of the X-ray and optical/UV orbital ephemeris of X1822-371
}

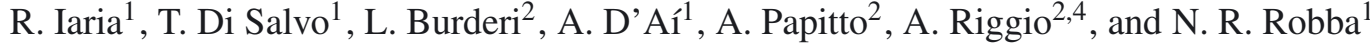 \\ ${ }^{1}$ Dipartimento di Fisica, Università di Palermo, via Archirafi 36, 90123 Palermo, Italy \\ e-mail: iaria@fisica.unipa.it \\ 2 Dipartimento di Fisica, Università degli Studi di Cagliari, SP Monserrato-Sestu, KM 0.7, 09042 Monserrato, Italy \\ 3 INAF, Istituto di Astrofisica Spaziale e Fisica cosmica di Palermo, via U. La Malfa 153, 90146 Palermo, Italy \\ ${ }^{4}$ INAF, Osservatorio Astronomico di Cagliari, Poggio dei Pini, Strada 54, 09012 Capoterra (CA), Italy
}

Received 24 May 2011 / Accepted 21 July 2011

\begin{abstract}
Aims. Recent studies of the optical/UV and X-ray ephemerides of X1822-371 have found some discrepancies in the value of the orbital period derivative. Because of the importance of this value in constraining the system evolution, we comprehensively analyse all the available optical/UV/X eclipse times of this source to investigate the origin of these discrepancies.

Methods. We collected all previously published X-ray eclipse times from 1977 to 2008, to which we added the eclipse time observed by Suzaku in 2006. This point is very important to cover the time gap between the last RXTE eclipse time (taken in 2003) and the most recent Chandra eclipse time (taken in 2008). Similarly we collected the optical/UV eclipse arrival times covering the period from 1979 to 2006, adding a further eclipse time taken on 1978 and updating previous optical/UV ephemeris. We compared the X-ray and the optical/UV ephemeris, and finally derived a new ephemeris of the source by combining the eclipse arrival times in the X-ray and optical/UV bands.

Results. The X-ray eclipse time delays calculated with respect to a constant orbital period model display a clear parabolic trend, confirming that the orbital period of this source constantly increases at a rate of $\dot{P}_{\text {orb }}=1.51(7) \times 10^{-10} \mathrm{~s} / \mathrm{s}$. Combining the X-ray and the optical/UV data sets, we find that $\dot{P}_{\text {orb }}=1.59(9) \times 10^{-10} \mathrm{~s} / \mathrm{s}$, which is compatible with the X-ray orbital solution. We also investigate the possible presence of a delay of the optical/UV eclipse with respect to the X-ray eclipse, finding that this delay may not be constant in time. In particular, this variation is compatible with a sinusoidal modulation of the optical/UV eclipse arrival times with respect to the long-term parabolic trend. In this case, the optical/UV eclipse should lag the X-ray eclipse and the time-lag oscillate about an average value.

Conclusions. We confirm that the orbital period derivative is three orders of magnitude larger than expected from conservative mass transfer driven by magnetic braking and gravitational radiation.
\end{abstract}

Key words. stars: neutron - X-rays: binaries - X-rays: stars - stars: individual: X1822-371

\section{Introduction}

$\mathrm{X} 1822-371$ is an eclipsing compact binary system with a period of $5.57 \mathrm{~h}$ hosting a $0.59 \mathrm{~s}$ X-ray pulsar. Several authors have reported new orbital ephemeris of the source using observations performed in different energy bands. Burderi et al. (2010, hereafter BU10) analysed X-ray data of X1822-371 covering the period from 1996 to 2008 to determine the eclipse times of the source and improved the previous X-ray ephemeris of X1822-371 reported by Parmar et al. (2000, hereafter PA00) that covered the period from 1977 to 1996 . BU10 added their data to those used by PA00 finding a positive derivative of the orbital period of $(1.499 \pm 0.071) \times 10^{-10} \mathrm{~s} / \mathrm{s}$ that is compatible with the previous one given by PA00 but with a smaller associated error.

Bayless et al. (2010, hereafter BA10) obtained the opti$\mathrm{cal} / \mathrm{UV}$ ephemeris of X1822-371 using data covering the period from 1979 to 2006 . They obtained a value of the orbital period derivative of $(2.12 \pm 0.18) \times 10^{-10} \mathrm{~s} / \mathrm{s}$, which is compatible with that reported by PA00 but slightly larger than the value proposed by BU10.

Ji et al. (2011, hereafter JI11), using the X-ray eclipse arrival times reported by PA00 and the eclipse arrival times inferred by the two Chandra/HETG observations of X1822-371 performed in 2000 (Obs ID: 671) and in 2008 (Obs ID: 9076 and 9858), already included in the work of BU10, estimated a value of the orbital period derivative of $(0.83 \pm 0.16) \times 10^{-10} \mathrm{~s} / \mathrm{s}$, with the error at the $90 \%$ confidence level, almost a factor of two smaller than the value reported by BU10.

We summarise the values of the eclipse reference time $T_{0}^{\mathrm{e}}$, the orbital period $P_{\text {orb } 0}$, and the orbital period derivative $\dot{P}_{\text {orb }}$ obtained by PA00, BU10, BA10, and J111 in Table 1.

In this work, we comprehensively examine both X-ray and optical/UV eclipse arrival times to give the most updated ephemeris of X1822-371, adding to the eclipse arrival times reported by BU10 the one obtained from a Suzaku observation performed in 2006. We also include a data point from a Ginga observation performed in 1989, and a data point from a ROSAT observation performed in 1992. We critically examine the discrepancies that have emerged in calculating the orbital ephemeris in previous papers and, finally, show the ephemeris of the X1822-371 by combining the optical/UV and X-ray data-sets.

\section{Suzaku observation}

Suzaku observed X1822-371 on 2006 October 2 with an elapsed time of $88 \mathrm{ks}$, the start and stop times of the observation corresponding to 54010.48 and 54011.50 MJD, respectively. 
Table 1. Journal of the ephemerides of X1822-371 discussed in this work.

\begin{tabular}{lcccc}
\hline \hline Parameters & Parmar et al. (2000) & Burderi et al. (2010) & Bayless et al. (2010) & Ji et al. (2011) \\
\hline$T_{0}^{\mathrm{e}}\left(\mathrm{MJD}_{\odot}\right)$ & $45614.80964(15)$ & $45614.80948(14)$ & $45614.81166(74)$ & $45614.80927(25)$ \\
$P_{\text {orb } 0}(\mathrm{~s})$ & $20054.1990(43)$ & $20054.2056(22)$ & $20054.1866(69)$ & $20054.2181(41)$ \\
$\dot{P}_{\text {orb }}\left(\times 10^{-10} \mathrm{~s} / \mathrm{s}\right)$ & $1.78(20)$ & $1.499(71)$ & $2.12(18)$ & $0.827(95)$ \\
$\chi^{2} /($ d.o.f. $)$ & $21.4 / 16$ & $38.69 / 25$ & $70.04 / 32$ & $35.99 / 19$ \\
\hline
\end{tabular}

Notes. Uncertainties are at the $68 \%$ c.l. for a single parameter. We show the reference time $T_{0}^{\mathrm{e}}$ of the eclipse arrival times in units of MJD, the orbital period $P_{\text {orb } 0}$ in units of seconds calculated at $T_{0}^{\mathrm{e}}$, the derivative of the orbital period $\dot{P}_{\text {orb }}$ in units of s/s, and finally the $\chi^{2} /($ d.o.f.) obtained fitting the eclipse arrival times with a quadratic function.

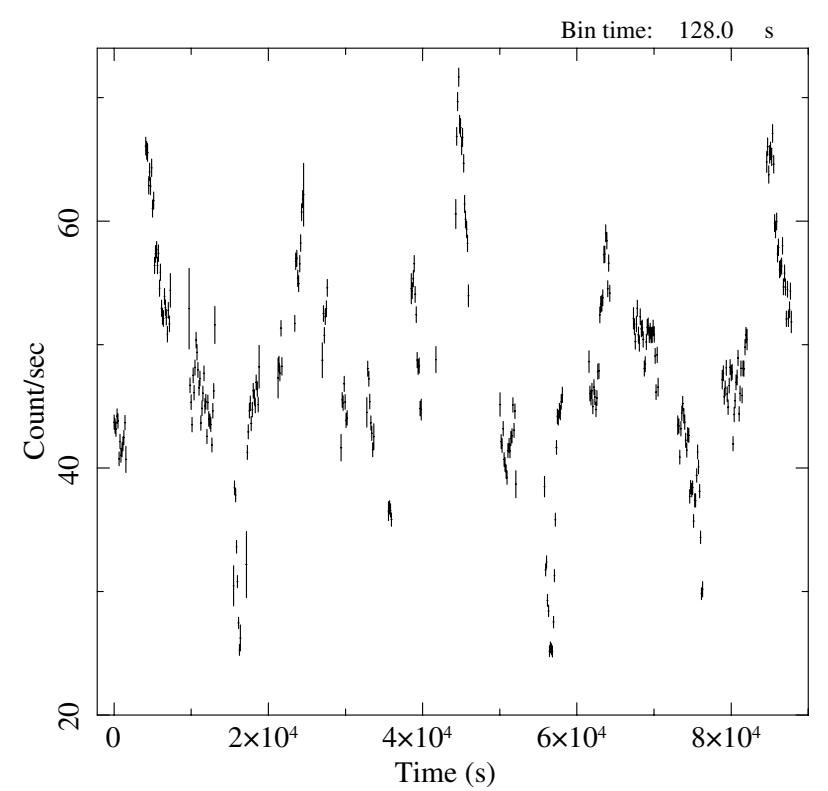

Fig. 1. Combined XIS light curve of X1822-371 in the $1-10 \mathrm{keV}$ energy band. The adopted bin time is $128 \mathrm{~s}$.

Both the X-ray Imaging Spectrometer (0.2-12 keV, XIS; Koyama et al. 2007) and the Hard X-ray Detector (10-600 keV, HXD; Takahashi et al. 2007) instruments were used during these observations. In this work, we used only the XIS data. There are four XIS detectors, numbered 0 to 3. The XIS0, XIS2, and XIS3 detectors use front-illuminated CCDs and have very similar responses, while XIS1 uses a back-illuminated CCD.

We reprocessed the observation using the aepipeline tool included in the Suzaku FTOOLS Version 16 applying the latest calibration available as of 2011 March. During the observation, XIS0 and XIS1 were used adopting the quarter window option (frame time $2 \mathrm{~s}$ ), while XIS2 and XIS3 worked in full window (frame time $8 \mathrm{~s}$ ) mode. We barycentred the XIS data using the Suzaku tool aebarycen and adopting as the best estimate of the source coordinates those derived from the 2008 Chandra observations (RA: 1825 46.81, Dec: -37 06 18.5, uncertainty: 0.6").

We extracted the four XIS light curves in the $1-10 \mathrm{keV}$ energy band selecting a circular region centred on the source. We adopted a radius of 130 pixels for XIS0 and XIS1 and 160 pixels for XIS2 and XIS3. The four light curves are quite similar and enclose four orbital periods of X1822-371, thus we used the FTOOL lcmath to combine the four XIS light curves. The combined XIS light curve is shown in Fig. 1 adopting a bin time of $128 \mathrm{~s}$

During only the third orbital passage of X1822-371, the eclipse was fully covered by Suzaku at a time of $55000 \mathrm{~s}$ from
Table 2. Journal of all the available X-ray eclipse times.

\begin{tabular}{lcccc}
\hline \hline Eclipse time $\left(\mathrm{JD}_{\odot}\right)$ & Error & Cycle & Ref. & Satellite \\
\hline 2443413.5272 & 0.0046 & -9486 & 1 & HEAO-1 Scan \\
2443591.5521 & 0.0046 & -8719 & 1 & HEAO-1 Scan \\
2443776.5459 & 0.0012 & -7922 & 1 & HEAO-1 Point \\
2443778.4065 & 0.0046 & -7914 & 1 & HEAO-1 Scan \\
2443969.4247 & 0.0069 & -7091 & 2 & Einstein \\
2444133.5277 & 0.0030 & -6384 & 1 & Einstein \\
2445580.4932 & 0.0005 & -150 & 1 & EXOSAT \\
2445615.30940 & 0.00038 & 0 & 1 & EXOSAT \\
2445963.00914 & 0.00033 & 1498 & 1 & EXOSAT \\
2445963.24046 & 0.00030 & 1499 & 1 & EXOSAT \\
2445963.47254 & 0.00034 & 1500 & 1 & EXOSAT \\
2446191.63643 & 0.00031 & 2483 & 1 & EXOSAT \\
2446191.86768 & 0.00033 & 2484 & 1 & EXOSAT \\
2446192.10008 & 0.00029 & 2485 & 1 & EXOSAT \\
2447760.22900 & 0.00030 & 9241 & 1 & Ginga \\
2448692.84396 & 0.00070 & 13259 & 1 & ROSAT \\
2449268.00984 & 0.00040 & 15737 & 3 & ASCA \\
2450353.35425 & 0.00035 & 20413 & 3 & ASCA \\
2450353.58728 & 0.00023 & 20414 & 3 & RXTE \\
2450701.51870 & 0.00120 & 21913 & 3 & BeppoSAX \\
2450992.58580 & 0.00230 & 23167 & 4 & RXTE \\
2451780.13170 & 0.00190 & 26560 & 4 & Chandra \\
2451975.56934 & 0.00056 & 27402 & 4 & XMM-Newton \\
2451975.56935 & 0.00031 & 27402 & 4 & RXTE \\
2452432.59458 & 0.00030 & 29371 & 4 & RXTE \\
2452488.53300 & 0.00038 & 29612 & 4 & RXTE \\
2452519.63569 & 0.00085 & 29746 & 4 & RXTE \\
2452882.65470 & 0.00037 & 31310 & 4 & RXTE \\
2454011.17300 & 0.00090 & 36172 & 5 & Suzaku \\
2454607.69592 & 0.00056 & 38742 & 4 & Chandra \\
\hline & & & &
\end{tabular}

References. (1) Hellier \& Smale 1994; (2) Hellier \& Mason 1989; (3) Parmar et al. 2000; (4) Burderi et al. 2010; (5) this work. The number of cycles for each eclipse time is discussed in Sect. 3.1.

the start time. To estimate the eclipse arrival time, we folded the combined XIS light curve, adopting the ephemeris reported by BU10 and a bin time of $128 \mathrm{~s}$. We fitted the orbital light curve to derive eclipse arrival times by adopting the same procedure described in BU10, obtaining an eclipse time passage at $54010.6730 \pm 0.0009 \mathrm{MJD}_{\odot}$ with an associated error at the $68 \%$ confidence level.

\section{The ephemeris of $\mathrm{X} 1822-371$}

For clarity's sake, we show in Table 2 the X-ray eclipse arrival times that we used to update the X-ray ephemeris of X1822371. Most of these data points were included in the timing analysis of BU10. To their data set, we added eclipse arrival times from Ginga (1989), ROSAT (1992), and, most impor- 


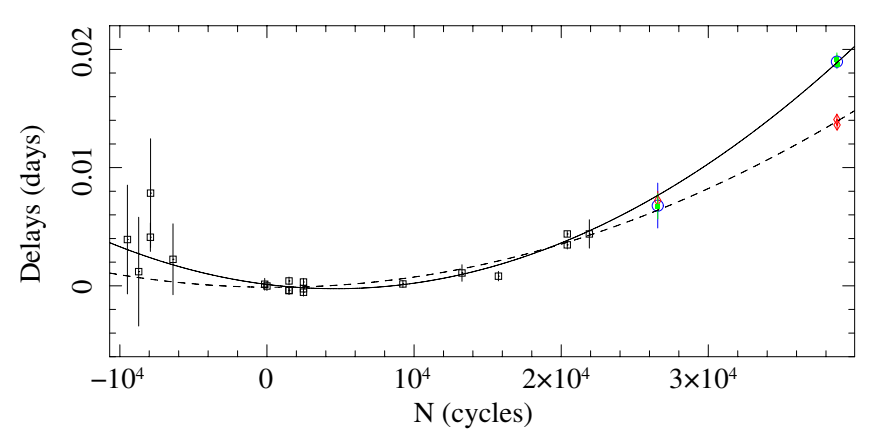

Fig. 2. Time delays of the eclipse arrival times with respect to the linear ephemeris of Hellier \& Smale (1994). The black open squares correspond to the eclipse arrival times shown by Hellier \& Smale (1994) and PA00, the red diamonds to the Chandra eclipse arrival times given by JI11, the green filled squares to the Chandra eclipse arrival times reported in this paper (see Table 3), the blue open circles to the Chandra eclipse arrival times reported by BU10. The dashed and solid lines are the best-fit quadratic curve obtained by JI11 and in this paper (see text), respectively. (This figure is available in color in the electronic form.)

tantly, Suzaku (2006). The ephemerides showed in Table 1 and in the analysis now described are given in barycentric dynamical time. We note that the RXTE arrival times from 1998 to 2003 reported in Table 1 of BU10 (except for the second point corresponding to cycle 23167 ) are not the eclipse arrival times, as erroneously stated, but the times of passage through the ascending node (which differs from the eclipse time by $P_{\text {orb }} / 4$ ). Nevertheless, the corresponding RXTE time delays were correctly shown in Fig. 1 of BU10 and correctly used to derive the orbital ephemeris, which are therefore unaffected by this mistake. The correct RXTE eclipse arrival times are shown in our Table 2. The X-ray ephemeris of X1822-371 reported by BU10 and JI11 show a large discrepancy in the quadratic term by almost a factor of two. JI11 suggested that the discrepancy in the time delay associated with the last two Chandra observations is caused by BU10 not folding the Chandra light curves to estimate the eclipse arrival time, which instead was done by BU10. To understand the reason for this discrepancy, as a first step we tried to reproduce the results of JI11 by using the same data they used in their analysis. These consist of a total of 22 eclipse-times, which are, respectively, those given by Hellier \& Smale (1994), PA00, and three eclipse arrival times obtained from three Chandra observations corresponding to obsID 671, 9076, and 9058 derived by JI11 (see Table 2 in their paper).

We found the corresponding time delays following their procedure, namely we determined the time delays with respect to the best-fit linear ephemeris shown by Hellier \& Smale (1994), that is

$$
T_{\text {ecl }}=2445615.30942(14) \mathrm{JD}_{\odot}+0.232109017(33) N,
$$

and fitted the time delays with a quadratic function. We obtained best-fit values consistent with the ones reported in JI11. We showed in Fig. 2 the time delays in units of days associated with the eclipse arrival times shown by Hellier \& Smale (1994) and PA00 with black open squares. The delay times associated with the Chandra eclipse arrival times showed by JI11 were plotted using red diamonds. The dashed line corresponds to the quadratic best-fit curve given by JI11.

After establishing the reproducibility of the parameter's estimates of JI11, we explored the cause of the discrepancy in the fitting results given by BU10 extracting the eclipse arrival times from each Chandra barycentred and folded light curve
Table 3. Eclipse arrival times of the three Chandra observations.

\begin{tabular}{lccc}
\hline \hline ObsID & Eclipse time $\left(\mathrm{MJD}_{\odot}\right)$ & Cycle & Delay (days) \\
\hline 671 & $51779.8638(11)$ & 26561 & $0.0067(11)$ \\
9076 & $54607.19610(56)$ & 38742 & $0.01914(56)$ \\
9858 & $54609.74890(31)$ & 38753 & $0.01875(31)$ \\
\hline
\end{tabular}

Notes. Column 1: Chandra obsIDs. Column 2: our estimation of the eclipse arrival time. Column 3: the corresponding cycle with respect to the linear ephemeris given by Hellier \& Smale (1994). Column 4: the corresponding delay in units of days. The errors are at the $68 \%$ c.l.

(obsIDs 671, 9076, and 9858). We show the Chandra eclipse arrival times in Table 3 . The corresponding delays were estimated as described above.

We found that the discrepancies of the eclipse arrival times between our analysis and JI11's are $-40 \pm 140 \mathrm{~s}, 440 \pm 70 \mathrm{~s}$, and $440 \pm 50 \mathrm{~s}$ for obsIDs 671,9076 , and 9858 , respectively. Since the errors are at the $68 \%$ c.l., the eclipse arrival times corresponding to the obsIDs 9076 and 9858 are not compatible. We show our Chandra delays with green filled squares in Fig. 2. Only two eclipse times were derived by BU10 from the Chandra observations, because light curves of obsID 9076 and obsID 9058 were combined to obtain a single folded light-curve and a single eclipse-time passage, with a smaller uncertainty, since the observations were sufficiently close in time to each other. We show the two corresponding time delays with blue open circles in Fig. 2. We note that our delays and those given by BU10 are widely compatible.

Fitting the time delays corresponding to the eclipse arrival times given by Hellier \& Smale (1994), PA00, and our three eclipse arrival times reported in Table 3, for a total of 22 data points, we obtained

$$
\begin{aligned}
T_{\mathrm{ecl}}=45614.80954(14) \mathrm{MJD}_{\odot}+ & 0.2321088628(21) N \\
+ & 1.648(72) \times 10^{-11} N^{2},
\end{aligned}
$$

with a $\chi^{2} /$ (d.o.f.) $=25.6 / 19$ and the errors are at the $68 \%$ confidence level, the uncertainties in the parameters have been scaled by a factor $\sqrt{\chi_{\text {red }}^{2}}$ to take into account a $\chi_{\text {red }}^{2}$ of the bestfit model larger than 1 . We note that the quadratic term is larger than that shown by JI11. The corresponding $P_{\text {orb } 0}$ and $\dot{P}_{\text {orb }}$ are $20054.20574(17) \mathrm{s}$ and $1.420(63) \times 10^{-10} \mathrm{~s} / \mathrm{s}$, respectively. These values are compatible within one $\sigma$ with the ones given by BU10 (see Table 1).

\subsection{Updated X-ray ephemeris of $X 1822-371$}

As a first step, we found the X-ray ephemeris of X1822-371 using the eclipse arrival times adopted by BU10 excluding the Chandra eclipse arrival times and including the eclipse arrival times taken with Ginga, ROSAT, and Suzaku (see Table 2) for a total of 28 available data points. The Suzaku data-point is very important in this respect, since it was taken in 2006 and therefore precedes the last Chandra data-points taken in 2008. This is very important to fill the time gap between the last RXTE arrival time taken in 2003 and the most recent Chandra observation taken in 2008, and therefore gives us the opportunity to discriminate more clearly between the Chandra eclipse arrival time as reported by JI11 and our measurement (which is compatible with the one reported by BU10).

We found the delays of the eclipse arrival times by subtracting from our measurements the eclipse arrival times predicted 


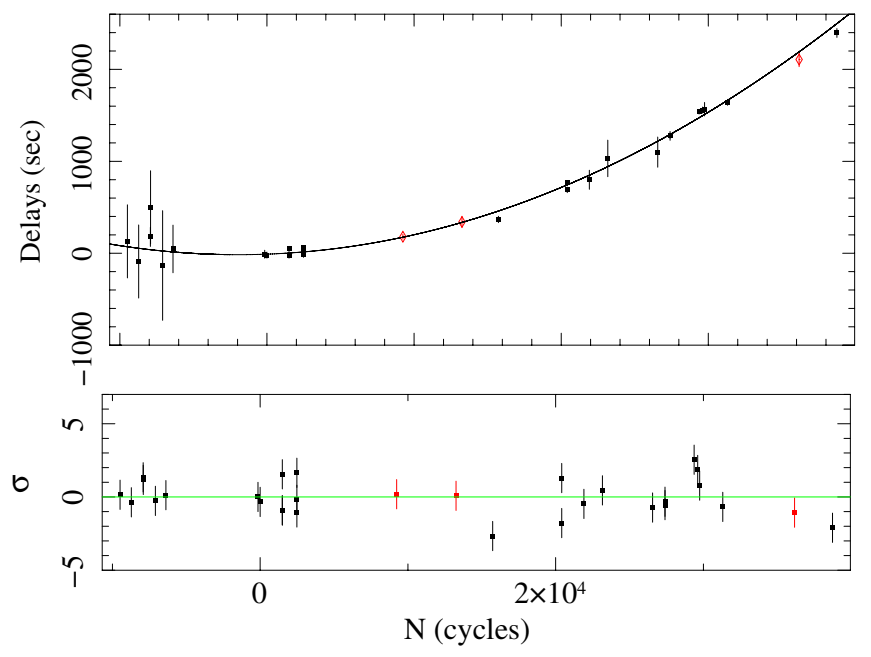

Fig. 3. Upper panel: eclipse time delays with respect to a constant orbital period model plotted versus the orbital cycle for all the available $\mathrm{X}$-ray eclipse time measures together with the best-fit parabola. Lower panel: residuals in units of $\sigma$ with respect to the best-fit parabola. The black full squares points are from BU10, the red diamonds are the data added in this work. (This figure is available in color in the electronic form.)

by a constant orbital period model adopting the orbital period, $P_{\text {orb } 0}$, and the reference time, $T_{0}^{\mathrm{e}}$, given by PA00. The time delays were plotted versus the orbital cycle number $N$. The integer $N$ is the exact number of orbital cycles elapsed since $T_{0}^{\mathrm{e}}$; the cycle number $N$ corresponding to each eclipse arrival time is shown in Col. 3 of Table 2. We then fitted the time delays using a parabolic function obtaining a $\chi^{2} /$ (d.o.f.) of $33.63 / 25$. We found that $T_{0}^{\mathrm{e}}=45614.80959(16) \mathrm{MJD}_{\odot}, P_{\text {orb } 0}=20054.2020(28)$ s, and $\dot{P}_{\text {orb }}=1.626(90) \times 10^{-10} \mathrm{~s} / \mathrm{s}$ with the associated errors at the $68 \%$ confidence level. All these values are compatible within one $\sigma$ with those given by BU10 (see Col. 3 in Table 1), this suggests that the Chandra eclipse arrival times given by BU10 are in agreement with all the previous points.

To update the X-ray ephemeris of X1822-371, we then included the Chandra eclipse arrival times given by BU10 in our data set for a total of 30 available data points. We found the corresponding delays and cycle numbers as described above. The time delays are shown in the upper panel of Fig. 3. We plotted the time delays used by BU10 with black full squares, while the time delays added in this work and corresponding to the Ginga, ROSAT, and Suzaku eclipse times are shown with red diamonds. We then fitted the time delays using a parabolic function, resulting in the ephemeris

$$
\begin{aligned}
T_{\mathrm{ecl}}=45614.80953(16) \mathrm{MJD}_{\odot}+ & 0.232108853(30) N \\
+ & 1.757(93) \times 10^{-11} N^{2},
\end{aligned}
$$

where the associated errors are at the $68 \%$ confidence level. We obtained a $\chi^{2} /$ (d.o.f.) of $41.2 / 27$, the best-fit curve is shown with a solid line in Fig. 3. We show the residuals in units of $\sigma$ in the lower panel of Fig. 3 and report the obtained values of $T_{0}^{\mathrm{e}}, P_{\text {orb } 0}$, and $\dot{P}_{\text {orb }}$ in the second column of Table 4 .

We found that the derivative of the orbital period, $\dot{P}_{\text {orb }}$, is $1.514(80) \times 10^{-11} \mathrm{~s} / \mathrm{s}$, compatible with the value of $1.499(71) \times$ $10^{-11} \mathrm{~s} / \mathrm{s}$ estimated by BU10.
Table 4. Updated X-ray and optical/UV ephemeris of X1822-371.

\begin{tabular}{lcc}
\hline \hline Parameters & X-ray & Optical/UV \\
\hline$T_{0}^{\mathrm{e}}\left(\mathrm{MJD}_{\odot}\right)$ & $45614.80953(16)$ & $45614.8116(11)$ \\
$P_{\text {orb 0 }}(\mathrm{s})$ & $20054.2049(26)$ & $20054.188(10)$ \\
$\dot{P}_{\text {orb }}$ & $1.514(80)$ & $2.10(26)$ \\
$\chi^{2} /$ (d.o.f.) & $41.2 / 27$ & $71.38 / 33$ \\
\hline
\end{tabular}

Notes. Uncertainties are at the $68 \%$ c.l. for a single parameter. The parameters are defined as in Table 1 , the derivative of the orbital period is in units of $10^{-10} \mathrm{~s} / \mathrm{s}$. The updated values of $T_{0}^{\mathrm{e}}, P_{\text {orb } 0}$, and $\dot{P}_{\text {orb }}$ using $\mathrm{X}$-ray data and optical/UV data are shown in Cols. 2 and 3, respectively.

\subsection{Updated optical/UV ephemeris of X1822-371}

BA10 used 35 optical/UV eclipse arrival times shown in Table 1 of their paper to find the best-fit optical/UV ephemeris of $\mathrm{X} 1822-371$ given by

$$
\begin{aligned}
T_{\mathrm{ecl}}=45614.81166(74) \mathrm{MJD}+ & 0.232108641(80) \mathrm{N} \\
+ & 2.46(21) \times 10^{-11} N^{2},
\end{aligned}
$$

where the errors are at the $68 \%$ confidence level (Bayless, priv. comm.). We added to their data the optical eclipse arrival time $2443629.841 \pm 0.013 \mathrm{JD}_{\odot}$ given by Hellier \& Mason (1989) and not included in BA10.

Using the 36 optical/UV data points and following the procedure described in the previous section we found the corresponding time delays. Fitting them with a parabola, we obtained the following optical/UV ephemeris

$$
\begin{aligned}
T_{\mathrm{ecl}}=45614.8116(11) \mathrm{MJD}_{\odot}+ & 0.23210865(12) N \\
& +2.44(31) \times 10^{-11} N^{2},
\end{aligned}
$$

with a $\chi^{2} /($ d.o.f.) of $71.38 / 33$ and the errors are at the $68 \%$ confidence level. The uncertainties have been scaled by a factor $\sqrt{\chi_{\text {red }}^{2}}$ to take into account a $\chi_{\text {red }}^{2}$ of the best-fit model larger than 1 . This explains why the uncertainties in the optical/UV ephemeris we have shown are larger than the ephemeris shown by BA10. The updated optical/UV ephemeris are consistent with those given by BA10. We report the corresponding values of $T_{0}^{\mathrm{e}}, P_{\text {orb } 0}$, and $\dot{P}_{\text {orb }}$ in the third column of Table 4. In the upper panel of Fig. 4, we show the time delays for each eclipse arrival time of X1822371 for the X-ray (red full squares) and optical/UV bands (black full squares) for a total of 66 data points. The solid and dashed lines correspond to the best-fit parabolas reproducing the X-ray and optical/UV ephemerides showed in Eqs. (2) and (4), respectively.

We compare the X-ray and optical/UV residuals with the $\mathrm{X}$-ray best-fit parabola in Fig. 4 (middle panel). Although we plot the residuals for the best-fit parabola obtained from the $\mathrm{X}$-ray time delays, we note that almost all of the optical/UV data are close to the best-fit curve. The largest discrepancies are associated with the last two optical eclipse times shown by Hellier \& Mason (1989) corresponding to orbital cycles 7243 and 7600 and the two UV eclipse arrival times obtained with HST and reported by BA10; these last two data points are at orbital cycles 35387 and 35 395, respectively. All the other optical/UV points are within two $\sigma$ of the corresponding values of the best-fit X-ray ephemeris.

In Fig. 4 (bottom panel), we show the X-ray and optical/UV residuals with respect to the optical/UV best-fit curve. In this case, the X-ray data are mainly below the best-fit optical/UV parabola. 

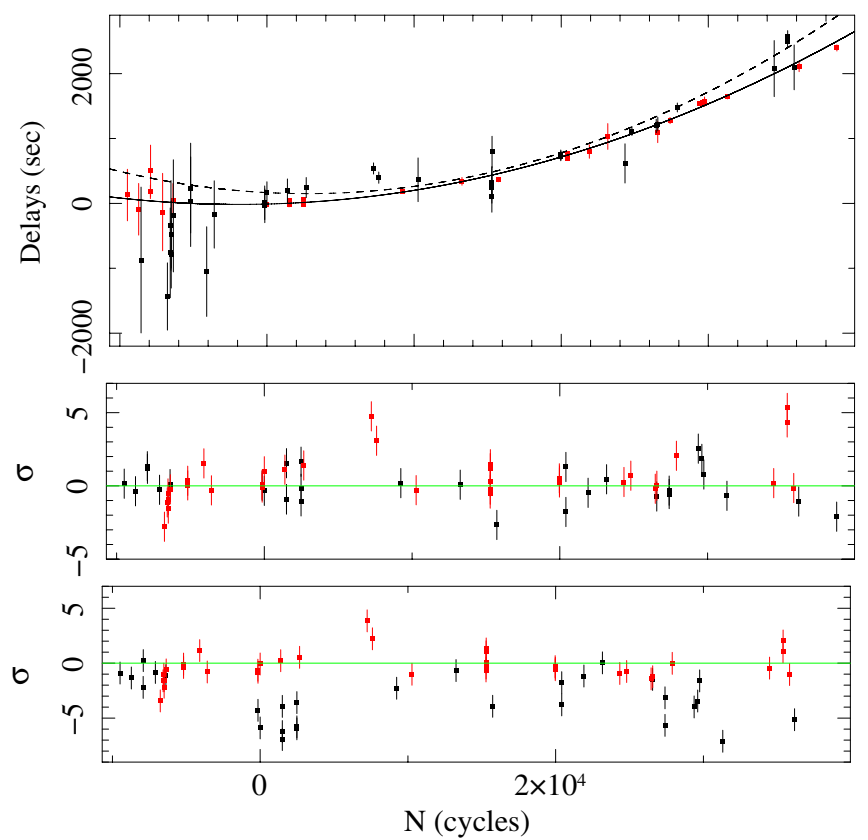

Fig. 4. Top panel: the optical/UV (black filled squares) and X-ray (red filled squares) time delays. The dashed and solid lines correspond to the optical/UV and X-ray best-fit parabolic curve. Middle panel: residuals with respect to the X-ray best-fit parabolic curve. Bottom panel: residuals with respect to the optical/UV best-fit parabolic curve. (This figure is available in color in the electronic form.)

\subsection{Time-lag between optical/UV and X-ray eclipse times}

To our knowledge, there are only two simultaneous X-ray and optical observations of the eclipse of X1822-371 reported by Hellier \& Mason (1989) and Hellier et al. (1990); these authors showed that the optical eclipse times lag the X-ray eclipse times by $3.0 \pm 3.4 \mathrm{~min}$, and $180 \pm 50 \mathrm{~s}$, respectively.

The optical eclipses are also wider than the X-ray eclipses; the different width suggests a different origin for the optical and X-ray eclipses, respectively. Hellier \& Mason (1989) proposed that the X-ray emission comes from an accretion disc corona (ADC) with a radius half of the outer accretion disc radius, while the optical emission is produced by a more extended disk structure. Furthermore, the optical eclipse lags the $\mathrm{X}$-ray eclipse because of the asymmetric disk structure probably caused by the stream impact onto the outer accretion disk. Hellier \& Mason (1989), modelling the X-ray and optical light curves of X1822-371, found an optical eclipse time-lag of $\sim 0.01$ in units of orbital phase, corresponding to a time-lag of $200 \mathrm{~s}$. BA10 discussed a marginally significant time-lag between the optical/UV and X-ray ephemeris of $100 \pm 65 \mathrm{~s}$ and $122 \mathrm{~s}$ with respect to the X-ray ephemeris reported by PA00 and BU10, respectively.

Since we have used an unprecedentedly large amount of optical/UV and X-ray eclipse times, we can estimate the average time-lag along 50000 orbital cycles with good accuracy. We fitted simultaneously the X-ray and optical/UV time delays allowing the constant terms of each parabola free to vary and constraining the values of the linear and quadratic parameters of each parabola to the same value, since the orbital period of X1822-371 and its derivative cannot depend on the considered waveband.

Fitting the time delays, we obtained a large $\chi^{2} /$ (d.o.f.) of $124.04 / 62$ and found that the best-fit values of the linear and quadratic terms are $(3.7 \pm 2.8) \times 10^{-3} \mathrm{~s}$ and $(1.595 \pm 0.086) \times 10^{-6} \mathrm{~s}$,
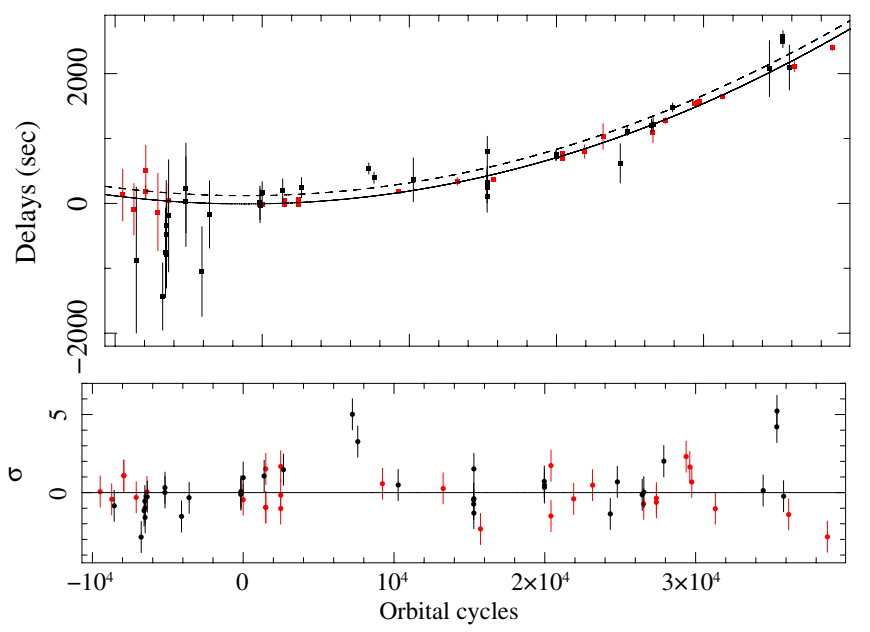

Fig. 5. Upper panel: the optical/UV (black filled squares) and X-ray (red filled squares) time delays fitted with two parabolas having the same linear and quadratic terms. The solid and dashed parabolas correspond to the X-ray and optical/UV best-fit curves. Lower panel: residuals in units of $\sigma$ with respect to the best-fit parabola describing the $\mathrm{X}$-ray ephemeris shown in Eq. (5). (This figure is available in color in the electronic form.)

respectively. The constant terms are $121 \pm 36 \mathrm{~s}$ and $-6 \pm 16 \mathrm{~s}$ for the optical/UV and X-ray data-sets, respectively. Using these values, we obtained the ephemerides for the X-ray and optical/UV data

$$
\begin{aligned}
T_{\mathrm{ecl}_{\mathrm{X}-\mathrm{ray}}}= & 45614.80957(19) \mathrm{MJD}_{\odot}+0.232108828(32) N \\
& +1.847(99) \times 10^{-11} N^{2} \\
T_{\mathrm{ecl}_{\mathrm{op} / \mathrm{UV}}}= & 45614.81104(42) \mathrm{MJD}_{\odot}+0.232108828(32) N \\
& +1.847(99) \times 10^{-11} N^{2} .
\end{aligned}
$$

The corresponding orbital period derivative is $1.591(86) \times$ $10^{-10} \mathrm{~s} / \mathrm{s}$, and the reference time $T_{0}$ is $45614.80957(19)$ and $45614.81104(42) \mathrm{MJD}_{\odot}$ for X-ray and optical/UV data-sets, respectively; all the errors are at the $68 \%$ c.l. For clarity's sake, the values of $T_{0}^{\mathrm{e}}, P_{\text {orb } 0}$, and $\dot{P}_{\text {orb }}$ are showed in Table 5. In the upper panel of Fig. 5, we show the X-ray (red points) and the optical/UV (black points) time delays; the dashed and solid curves are the optical/UV and X-ray best-fit parabolas, respectively.

From our analysis, we found a time-lag of $127 \pm 52 \mathrm{~s}$, which is significant at a confidence level of $2.4 \sigma$. In the bottom panel of Fig. 5, we show the residuals of the X-ray (red points) and optical/UV (black points) delays with respect to the X-ray bestfit parabola.

The values of the optical/UV time-lags with respect to the best-fit parabola describing the X-ray ephemeris given by Eq. (5) are shown in Table 6 . We note that the largest optical time-lags are associated with the last two optical eclipse times shown by Hellier \& Mason (1989) corresponding to orbital cycles 7243 and 7600 and with the recent two UV eclipse arrival times obtained with HST and reported by BA10; the corresponding timelags are $433 \pm 86 \mathrm{~s}, 282 \pm 86 \mathrm{~s}, 364 \pm 86 \mathrm{~s}$, and $452 \pm 86 \mathrm{~s}$, respectively. These values are larger than $200 \mathrm{~s}$, in disagreement with that predicted by Hellier \& Mason (1989) modelling the X-ray and optical light curves of X1822-371.

We fitted the optical/UV time-lags with a constant obtaining a large $\chi^{2}$ (d.o.f.) of 81.25(35); the constant value was $127 \mathrm{~s}$, which is similar to the averaged time-lag previously discussed. 
Table 5. Ephemeris of X1822-371 fitting simultaneously X-ray and optical/UV data.

\begin{tabular}{lcccc}
\hline \hline $\begin{array}{l}T_{0_{\mathrm{X} \text {-ray }}}^{\mathrm{e}} \\
\left(\mathrm{MJD}_{\odot}\right)\end{array}$ & $\begin{array}{c}T_{0_{\text {optical } / U V}}^{\mathrm{e}} \\
\left(\mathrm{MJD}_{\odot}\right)\end{array}$ & $\begin{array}{c}P_{\text {orb } 0} \\
(\mathrm{~s})\end{array}$ & $\begin{array}{c}\dot{P}_{\text {orb }} \\
\left(\times 10^{-10}\right) \mathrm{s} / \mathrm{s}\end{array}$ & $\chi^{2} /($ d.o.f. $)$ \\
\hline $45614.80957(19)$ & $45614.81104(42)$ & $20054.2027(28)$ & $1.591(86)$ & $124.04 / 62$ \\
\hline
\end{tabular}

Notes. Uncertainties are at $68 \%$ c.l. for a single parameter. The parameters are defined as in Table 1. The uncertainties in the parameters have been scaled by a factor $\sqrt{\chi_{\text {red }}^{2}}$ to take into account a $\chi_{\text {red }}^{2}$ of the best-fit model larger than 1 .

Table 6. Journal of the optical/UV time-lags.

\begin{tabular}{|c|c|c|c|}
\hline $\begin{array}{l}\text { Eclipse time } \\
\left(\mathrm{JD}_{\odot}-2400000\right)\end{array}$ & $\begin{array}{l}\text { Time-lag } \\
(\mathrm{s})\end{array}$ & $\begin{array}{c}\text { Residuals best-fit } 1 \\
\text { (s) }\end{array}$ & $\begin{array}{c}\text { Residuals best-fit } 2 \\
\text { (s) }\end{array}$ \\
\hline $43629.8410 \pm 0.0130$ & $-951 \pm 1123$ & $-938 \pm 1123$ & $-1278 \pm 1123$ \\
\hline $44044.8450 \pm 0.0060$ & $-1477 \pm 518$ & $-1444 \pm 518$ & $-1395 \pm 518$ \\
\hline $44090.1140 \pm 0.0080$ & $-800 \pm 691$ & $-767 \pm 691$ & $-644 \pm 691$ \\
\hline $44101.0280 \pm 0.0080$ & $-377 \pm 691$ & $-344 \pm 691$ & $-241 \pm 691$ \\
\hline $44105.6650 \pm 0.0060$ & $-824 \pm 518$ & $-791 \pm 518$ & $-701 \pm 518$ \\
\hline $44106.5970 \pm 0.0060$ & $-516 \pm 518$ & $-483 \pm 518$ & $-396 \pm 518$ \\
\hline $44137.9350 \pm 0.0100$ & $-227 \pm 864$ & $-195 \pm 864$ & $-252 \pm 864$ \\
\hline $44411.1320 \pm 0.0080$ & $218 \pm 691$ & $242 \pm 691$ & $247 \pm 691$ \\
\hline $44412.0580 \pm 0.0080$ & $8 \pm 691$ & $32 \pm 691$ & $32 \pm 691$ \\
\hline $44664.8120 \pm 0.0080$ & $-1057 \pm 691$ & $-1051 \pm 691$ & $-916 \pm 691$ \\
\hline $44783.8940 \pm 0.0060$ & $-172 \pm 518$ & $-179 \pm 518$ & $-542 \pm 518$ \\
\hline $45579.5650 \pm 0.0030$ & $16 \pm 259$ & $-114 \pm 259$ & $-163 \pm 259$ \\
\hline $45580.7250 \pm 0.0030$ & $-31 \pm 259$ & $-162 \pm 259$ & $-216 \pm 259$ \\
\hline $45615.3115 \pm 0.0020$ & $166 \pm 173$ & $-30 \pm 173$ & $-173 \pm 173$ \\
\hline $45937.7110 \pm 0.0021$ & $192 \pm 181$ & $-4 \pm 181$ & $-160 \pm 181$ \\
\hline $46234.5787 \pm 0.0018$ & $228 \pm 156$ & $-20 \pm 156$ & $-83 \pm 156$ \\
\hline $47296.4798 \pm 0.0010$ & $433 \pm 86$ & $80 \pm 86$ & $158 \pm 86$ \\
\hline $47379.3410 \pm 0.0010$ & $282 \pm 86$ & $-72 \pm 86$ & $25 \pm 86$ \\
\hline $47999.7674 \pm 0.0039$ & $163 \pm 337$ & $-160 \pm 337$ & $209 \pm 337$ \\
\hline $49163.0960 \pm 0.0028$ & $-114 \pm 242$ & $-253 \pm 242$ & $37 \pm 242$ \\
\hline $49164.0237 \pm 0.0028$ & $-178 \pm 242$ & $-317 \pm 242$ & $-25 \pm 242$ \\
\hline $49164.2542 \pm 0.0028$ & $-317 \pm 242$ & $-456 \pm 242$ & $-164 \pm 242$ \\
\hline $49165.1852 \pm 0.0028$ & $-96 \pm 242$ & $-234 \pm 242$ & $59 \pm 242$ \\
\hline $49166.1190 \pm 0.0028$ & $368 \pm 242$ & $229 \pm 242$ & $524 \pm 242$ \\
\hline $50250.5308 \pm 0.0008$ & $49 \pm 69$ & $65 \pm 69$ & $22 \pm 69$ \\
\hline $50250.7626 \pm 0.0008$ & $22 \pm 69$ & $38 \pm 69$ & $-3 \pm 69$ \\
\hline $50252.6196 \pm 0.0008$ & $33 \pm 69$ & $49 \pm 69$ & $18 \pm 69$ \\
\hline $51264.8446 \pm 0.0035$ & $-413 \pm 302$ & $-409 \pm 302$ & $-713 \pm 302$ \\
\hline $51373.7094 \pm 0.0008$ & $47 \pm 69$ & $40 \pm 69$ & $-32 \pm 69$ \\
\hline $51756.4577 \pm 0.0012$ & $-15 \pm 104$ & $-74 \pm 104$ & $78 \pm 104$ \\
\hline $51782.4542 \pm 0.0012$ & $3 \pm 104$ & $-60 \pm 104$ & $-34 \pm 104$ \\
\hline $52089.7692 \pm 0.0008$ & $139 \pm 69$ & $24 \pm 69$ & $-40 \pm 69$ \\
\hline $53618.6767 \pm 0.0050$ & $58 \pm 432$ & $-285 \pm 432$ & $-134 \pm 432$ \\
\hline $53828.9720 \pm 0.0010$ & $364 \pm 86$ & $11 \pm 86$ & $11 \pm 86$ \\
\hline $53830.8299 \pm 0.0010$ & $452 \pm 86$ & $99 \pm 86$ & $95 \pm 86$ \\
\hline $53932.7201 \pm 0.0040$ & $-80 \pm 346$ & $-434 \pm 346$ & $-93 \pm 346$ \\
\hline
\end{tabular}

Notes. The optical/UV eclipse times (1st column), the corresponding time-lags (2nd column) with respect to the best-fit parabola describing the $\mathrm{X}$-ray ephemeris showed in Eq. (5), the time-lags after removal of the sinusoidal modulations (3rd and 4th column, respectively). The errors are at the $68 \%$ c.l.

In Fig. 6 (top panel), we show the optical/UV time-lags as a function of time in units of days, the dashed line being the constant function. We note that if we remove the four optical/UV points mentioned above, we find no significant time-lag between the optical/UV and X-ray eclipse times, with a best-fit value of $31 \pm 46 \mathrm{~s}$.

Because we found a large value of $\chi_{\text {red }}^{2}$, after a visual inspection of the fit residuals, we decided to fit the time-lags with the function $f(t)=A-B \sin \left[2 \pi / P\left(t-t_{0}\right)\right]$. In this case, we largely improved the fit for two different sets of parameters. For the first set, we obtained a $\chi^{2}$ (d.o.f.) $=34.52(32)$ and a probability of chance improvement with respect to the fit with a constant of $4.03 \times 10^{-6}$. The values of the best-fit parameters are
$A=161 \pm 24 \mathrm{~s}, B=194 \pm 29 \mathrm{~s}, P=6593 \pm 452 \mathrm{~d}(18.1 \pm 1.2 \mathrm{yr})$, and $t_{0}=-1180 \pm 481 \mathrm{~d}$, the errors being at the $68 \% \mathrm{c} .1$. For the second set of parameters, we obtained a $\chi^{2}$ (d.o.f.) $=35.57(32)$ and a probability of chance improvement with respect to the fit with a constant of $6.45 \times 10^{-6}$; in this case, $A=105 \pm 24 \mathrm{~s}$, $B=-267 \pm 43 \mathrm{~s}, P=283.1 \pm 0.6 \mathrm{~d}$, and $t_{0}=239 \pm 16 \mathrm{~d}$, the errors being at the $68 \% \mathrm{c} .1$. The best-fit values of both the fits are shown in Table 7.

The best-fit curve corresponding to the first set of parameters (hereafter best-fit 1) is shown in Fig. 6 (top panel) with a solid line. In the bottom panel of Fig. 6, we show the residuals with respect to the sinusoidal modulation in units of $\sigma$. The 


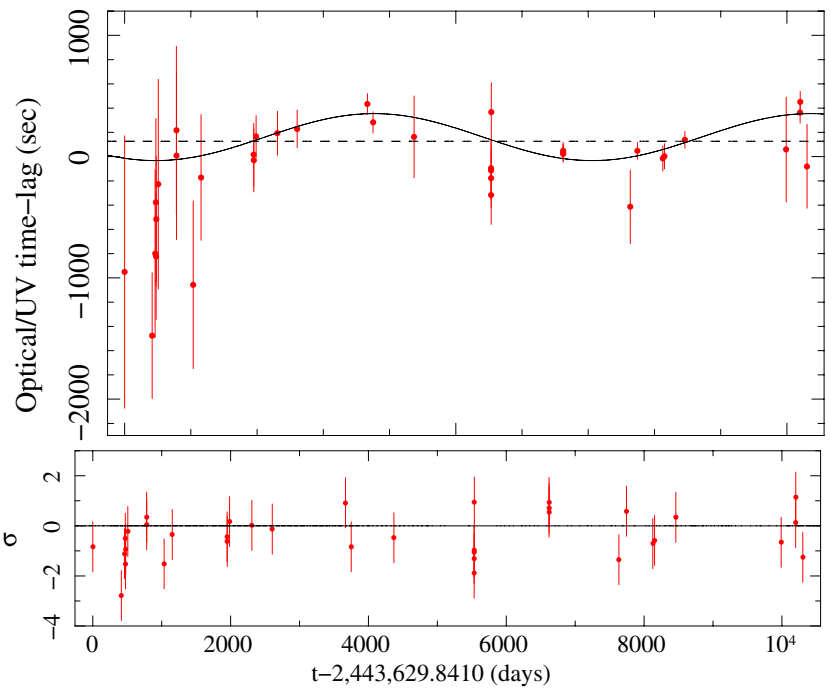

Fig. 6. Upper panel: the optical/UV (red filled squares) time-lags fitted with a constant (dashed line) and a sinusoidal function $f(t)$ (solid line) with a period of $18 \mathrm{yr}$ (see text). Lower panel: residuals in units of $\sigma$ with respect to the best-fit sinusoidal function. (This figure is available in color in the electronic form.)

Table 7. Best-fit parameters of the sinusoidal modulation fitting the optical/UV time-lags.

\begin{tabular}{lcc}
\hline \hline Parameters & Best-fit 1 & Best-fit 2 \\
\hline $\mathrm{A}(\mathrm{s})$ & $161 \pm 24$ & $105 \pm 24$ \\
$\mathrm{~B}$ (s) & $194 \pm 25$ & $-267 \pm 43$ \\
$\mathrm{P}$ (days) & $6593 \pm 452$ & $283.1 \pm 0.6$ \\
$t_{0}$ (days) & $-1180 \pm 481$ & $239 \pm 16$ \\
$\chi^{2}$ (d.o.f.) & $34.52(32)$ & $35.57(32)$ \\
\hline
\end{tabular}

Notes. Uncertainties are at the $68 \%$ c.l. for a single parameter. In the second and third columns, we show the best-fit parameters for the modulation of 18 years and 283 days, respectively.

values of the residuals corresponding to the first set of parameters are shown in the third column of Table 6 . The best-fit curve corresponding to the second set of parameters (hereafter bestfit 2) is shown in Fig. 7 (top panel) with a solid line. In the bottom panel of Fig. 7, we show the residuals with respect to the sinusoidal modulation in units of $\sigma$. The values of the residuals corresponding to the second set of parameters are shown in the fourth column of Table 6 . We checked our results fitting the $\mathrm{X}$-ray time-lags with respect to the best-fit parabola giving the $\mathrm{X}$-ray ephemeris. In this case, the residuals do not show any sinusoidal modulation, as expected. Fitting them with a constant, we found a $\chi^{2}$ (d.o.f.) $=42.8(29)$.

The two best-fit sinusoidal functions indicate that the optical/UV eclipse lags the X-ray eclipse with a time shift of either $161 \pm 24 \mathrm{~s}$ or $105 \pm 24$, which are significant at a confidence level of $6.7 \sigma$ and $4.4 \sigma$, respectively. These values are compatible with the time-lag predicted by Hellier \& Mason (1989), of 200 s. Our most intriguing result, never previously detected, is that the optical/UV eclipse times may oscillate in time with an amplitude of either $194 \pm 29$ s (best-fit 1) or $267 \pm 43$ s (best-fit 2), these values being significant at a confidence level of $6.7 \sigma$ and $6.2 \sigma$, respectively. The detected periods are $\sim 18 \mathrm{yr}$ (best-fit 1 ) and $\sim 283$ days (best-fit 2 ). Both the detected periods are very long and difficult to explain by invoking a superhump phenomenon (see Wang \& Chakrabarty 2010, and reference therein). The superhump excess $\epsilon$ is defined as $P_{\mathrm{sh}} / P_{\text {orb }}-1$, where $P_{\mathrm{sh}}$ is the
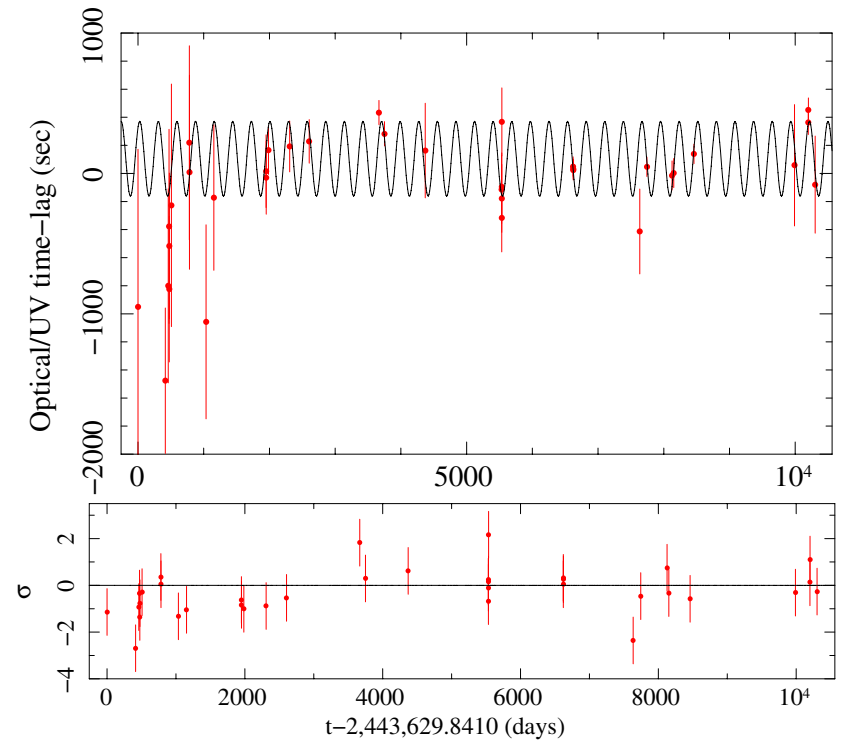

Fig. 7. Upper panel: the optical/UV (red filled squares) time-lags fitted with a sinusoidal function $f(t)$ (solid line) with a period of 283 days (see text). Lower panel: residuals in units of $\sigma$ with respect to the bestfit sinusoidal function. (This figure is available in color in the electronic form.)

superhump period, and also as $\epsilon=0.18 q+0.29 q^{2}$ (Patterson et al. 2005), where $q$ is the mass ratio $m_{2} / m_{1}$ with $m_{1}$ the neutron-star mass. Since the mass function and inclination angle of X1822-371 is well known (Jonker \& van der Klis 2001), assuming a neutron-star mass of $1.4 M_{\odot}$ and an inclination angle of $87^{\circ}$ we find that $q \simeq 0.29$ and $\epsilon \sim 0.077$. Consequently the superhump period should be $P_{\mathrm{sh}}=1.077 P_{\text {orb }}=21598.38 \mathrm{~s}$. A possible beat phenomenon between the superhump period and the orbital period could produce a period given by $\left(1 / P_{\mathrm{orb}}-1 / P_{\mathrm{sh}}\right)^{-1} \simeq$ 3.25 days. This value is shorter than the two periodicities that we reported.

We found that the lag changes with time and that this variation is compatible with a sinusoidal modulation at two different periods of $18 \mathrm{yr}$ and 283 days, respectively. However, we cannot exclude shorter periodicities, but our data set of only 36 optical/UV eclipse times spanning a time of 10000 days do not allow a rigorous study. To investigate this aspect, long optical/UV observations of X1822-371 covering several contiguous orbital periods of the source would be necessary.

Finally we note that both these best-fit curves are strongly driven by the optical measures corresponding to orbital cycles 7243 and 7600 and by the recent two UV eclipse arrival times obtained with HST and reported by BA10; these last two points are at orbital cycle 35387 and 35395.

\section{Conclusions}

We have revisited and discussed the X-ray and optical/UV ephemerides of X1822-371. Fitting simultaneously the optical/UV and X-ray time delays, we have found that the optical/UV eclipses of X1822-371 lag the X-ray eclipses by $127 \pm$ $52 \mathrm{~s}$ with a significance level of $2.4 \sigma$. However, this time-lag may not be constant in time. Fitting the optical/UV time-lags, we have found a statistically significant variation, which is compatible with a sinusoidal modulation at two different periods, $\sim 18 \mathrm{yr}$ and $239 \mathrm{~d}$. In the first case, the optical/UV eclipses lag the X-ray eclipse by an average time of $161 \mathrm{~s}$ (significance $6.7 \sigma$ ) and this delay oscillates in time around this value with an amplitude of 
$194 \mathrm{~s}$ (significance $6.7 \sigma$ ). In the second case, the optical/UV eclipses lag the X-ray eclipse by an average time of $105 \mathrm{~s}$ (significance $4.4 \sigma$ ), and this delay oscillates in time around this value with an amplitude of $267 \mathrm{~s}$ (significance $6.2 \sigma$ ).

Owing to the relatively small number of points over a longtime span of $30 \mathrm{yr}$, we cannot be sure of the period of this modulation, because we cannot exclude much shorter periods. Long and relatively continuous optical/UV observations are necessary to prove or disprove the presence of this periodicity in the optical/UV eclipse time-lags.

Our results confirm the value of the orbital solution derived by the X-ray eclipse times given by BU10 and that the orbital period derivative is three orders of magnitude larger than expected on the basis of the conservative mass transfer driven by magnetic braking and gravitational radiation. We have also confirmed this result by combining the X-ray data and the optical/UV data of X1822-371.

Acknowledgements. We thank the anonymous referee for the useful suggestions and A. Bayless for the fruitful interaction. authors acknowledge financial contribution from the agreement ASI-INAF I/009/10/0. A.P. acknowledges financial support from the Autonomous Region of Sardinia through a research grant under the program PO Sardegna ESF 2007-2013, L.R.7/2007, Promoting scientific research and technological innovation in Sardinia. L.B. and T.D. acknowledge support from the European Community's Seventh Framework Program (FP7/2007-2013) under grant agreement number ITN 215212 Black Hole Universe.

\section{References}

Bayless, A. J., Robinson, E. L., Hynes, R. I., Ashcraft, T. A., \& Cornell, M. E. 2010, ApJ, 709, 251

Burderi, L., di Salvo, T., Riggio, A., et al. 2010, A\&A, 515, A44

Hellier, C., \& Mason, K. O. 1989, MNRAS, 239, 715

Hellier, C., Mason, K. O., Smale, A. P., \& Kilkenny, D. 1990, MNRAS, 244 39P

Hellier, C., \& Smale, A. P. 1994, in The Evolution of X-ray Binariese, ed. S. Holt, \& C. S. Day, AIP Conf. Ser., 308, 535

Ji, L., Schulz, N. S., Nowak, M. A., \& Canizares, C. R. 2011, ApJ, 729, 102

Jonker, P. G., \& van der Klis, M. 2001, ApJ, 553, L43

Koyama, K., Tsunemi, H., Dotani, T., et al. 2007, PASJ, 59, 23

Parmar, A. N., Oosterbroek, T., Del Sordo, S., et al. 2000, A\&A, 356, 175

Patterson, J., Kemp, J., Harvey, D. A., et al. 2005, PASP, 117, 1204

Takahashi, T., Abe, K., Endo, M., et al. 2007, PASJ, 59, 35

Wang, Z., \& Chakrabarty, D. 2010, ApJ, 712, 653 